\title{
Structural Evidence of a Fourth Gla Residue in Fish Osteocalcin: Biological Implications $s^{\dagger, *}$
}

\author{
Carlos Frazão, ${ }^{*}$, Dina C. Simes,,${ }^{\prime}$ Ricardo Coelho,${ }^{\S}$ Dora Alves, ${ }^{\S}$ Matthew K. Williamson, ${ }^{\perp}$ Paul A. Price, ${ }^{\perp}$ \\ M. Leonor Cancela," and Maria A. Carrondo*,§ \\ ITQB-UNL, Av. da República, 2784-505 Oeiras, Portugal, CCMAR/University of Algarve-Campus de Gambelas, \\ 8005-139 Faro, Portugal, and Division of Biology, University of California at San Diego, La Jolla, California 92093
}

Received August 3, 2004; Revised Manuscript Received November 2, 2004

\begin{abstract}
Osteocalcin is a small (45 amino acids) secreted protein found to accumulate in bone and dentin of many organisms by interacting with calcium and hydroxyapatite, through the presence of three $\gamma$-carboxylated residues. In this work, we describe the first X-ray crystal structure for a nonmammalian osteocalcin, obtained at $1.4 \AA$ resolution, purified from the marine teleost fish Argyrosomus regius. The three-dimensional fit between the $A$. regius structure and that of the only other known X-ray structure, the porcine osteocalcin, revealed a superposition of the $\mathrm{C} \alpha$ atoms of their metal chelating residues, Gla and Asp, showing that their spatial distribution is consistent with the interatomic distances of calcium cations in the hydroxyapatite crystals. In both structures, the protein forms a tight globular arrangement of their three $\alpha$-helices while the remaining residues, at $\mathrm{N}$ - and $\mathrm{C}$-terminal regions, have essentially no secondary structure characteristics. This study revealed the presence of a fourth $\gamma$-carboxylation at Glu ${ }^{25}$, not previously detected in the structure of the porcine osteocalcin or in any other of the sequentially characterized mammalian osteocalcins (human, cow, and rat). A confirmation of the fourth Gla residue in A. regius osteocalcin was achieved via LC-MS analysis. These four doubly charged residues are, together with $\mathrm{Asp}^{24}$, concentrated in a common surface region located on the same side of the molecule. This further suggests that the known high affinity of osteocalcin for bone mineral may be derived from the clustering of calcium binding sites on this surface of the molecules.
\end{abstract}

Osteocalcin, also called bone Gla protein or BGP, ${ }^{1}$ was the first member of the calcium binding and vitamin K-dependent protein family not associated with blood coagulation to be described, sequenced, and characterized $(2,3)$. This small protein of 46-50 amino acid residues (depending on the species) $(4,5)$ is secreted mainly by osteoblasts. It is also one of the most abundant (10-20\%) noncollagenous proteins in bones of most vertebrates examined to date, from bony fish to mammals (depending on species, age, and site) (4, 6-9).

Conserved elements in all osteocalcin sequences examined to date include a single disulfide bond $\left(\mathrm{Cys}^{17}-\mathrm{Cys}^{23}\right)$ using Argyrosomus regius amino acid sequence numbering, and three $\gamma$-carboxyglutamic acid residues (Gla) located at positions 11,15 , and $18(4,9-13)$. These Gla residues are thought to facilitate adsorption of osteocalcin to hydroxy-

\footnotetext{
During part of this work, D.C.S. was the recipient of a PRODEP fellowship awarded by the Portuguese Ministry of Education. This work was partially supported by CCMAR funding to M.L.C. and D.C.S.

$\doteqdot$ Coordinates have been deposited in the Protein Data Bank (1) as entry $1 \mathrm{VZM}$.

* To whom correspondence should be addressed. Phone: +35121446967. Fax: +351-214433644. E-mail: carrondo@itqb.unl.pt, frazao@itqb.unl.pt.

$\S$ ITQB-UNL.

"CCMAR/University of Algarve.

${ }^{\perp}$ University of California at San Diego.

${ }^{1}$ Abbreviations: Gla, $\gamma$-carboxylated glutamic acid; LC-MS, liquid chromatography-mass spectrometry; BGP, bone Gla protein; FT-IRM, Fourier transform infrared microspectroscopy; $\mathrm{CD}$, circular dichroism; NMR, proton nuclear magnetic resonance.
}

apatite $(10,14,15)$ and are located in the central, conserved portion of the molecule. In contrast, its $\mathrm{N}$-terminal part exhibits considerable sequence variation $(4,8,9,13)$.

Initial studies showed that osteocalcin binds strongly to hydroxyapatite crystals (7) and affects mineral formation and mineral crystal growth in solution $(16,17)$. Several studies also suggested a role for osteocalcin as a matrix signal for the recruitment and differentiation of osteoclasts $(18-21)$. However, the understanding of the physiological function of osteocalcin was substantially advanced when a model for osteocalcin gene deficient mice was generated through mouse genetics (22). The osteocalcin "knockout" mice were characterized by a progressive increase in bone mass and accelerated rate of bone formation (22). Studies with this animal model provided evidence for osteocalcin as a negative regulator of bone formation without altering bone resorption or mineralization, although the precise mechanism by which osteocalcin exerted its control remained unclear. Subsequent studies on the osteocalcin knockout mice using Fourier transform infrared microspectroscopy (FT-IRM) indicated an apparent impaired bone maturation in these animals, implying a role for osteocalcin in stimulating bone mineral maturation (23). Despite this growing block of information, much remained to be understood concerning the molecular mechanism of action of this protein and the way it could interact in vivo with mineral ions and in particular with calcium.

Circular dichroism (CD) $(15,24,25)$ and ${ }^{1} \mathrm{H}$ NMR (24, 26) studies were performed to elucidate the structure of avian 
and mammalian osteocalcins in solution. They all agreed that the metal-free osteocalcin was essentially in a random coil conformation, but upon addition of millimolar levels of $\mathrm{Ca}^{2+}$, it shifted into an $\alpha$-helical structure.

The predicted secondary structure, calculated according to the definitions of Chou and Fasman (27) of chicken, several mammalian, and swordfish osteocalcins (15), gave common features since these species share extensive sequence identity. The model predicted two antiparallel $\alpha$-helical domains for the mammalian and chicken osteocalcins, several $\beta$-turns, and a $\beta$-sheet structure at the carboxyl terminus in all these species, and also at the amino terminus of mammalian osteocalcins. In swordfish, osteocalcin was predicted as a single long $\alpha$-helix that probably occupied positions 13-33 (15). In this model, all Gla side chains were placed on the same face of one $\alpha$-helix, spaced at intervals of $\sim 5.4 \AA$, closely paralleling the interatomic distance of $\mathrm{Ca}^{2+}$ atoms in the hydroxyapatite lattice (15).

Several other conformational studies of mammalian osteocalcins were directed toward the elucidation of the major structural elements of the protein in solution and its metal complexes. These were performed by using one-dimensional (1D) ${ }^{1} \mathrm{H},{ }^{113} \mathrm{Cd}$, and ${ }^{31} \mathrm{P}$ NMR (bovine) (28), 1D ${ }^{1} \mathrm{H}$ NMR (dog) (29), and 1D and two-dimensional (2D) ${ }^{1} \mathrm{H}$ NMR (rabbit) (24), but the structural information obtained was very limited. More recently, the three-dimensional structure of calcium-bound bovine osteocalcin was obtained using 2D ${ }^{1} \mathrm{H}$ NMR techniques that calculated a family of 13 structures (26). The tertiary structure of the protein consisted of an unstructured $\mathrm{N}$-terminus, a C-terminal loop, and several elements of secondary structure, including type III turns and two $\alpha$-helical regions. Also in this model, the three Gla residues were surface-exposed on the same side of the helical turns. The NMR-derived structure was proposed to bind three calcium atoms coordinated by oxygen atoms from its three Gla side chain $\mathrm{COOH}$ groups and one $\mathrm{COOH}$ Asp side chain. More recently, the X-ray crystal structure of the porcine osteocalcin at $2.0 \AA$ resolution (30) showed a negatively charged protein surface that coordinated five calcium ions in a spatial orientation similar to the calcium ion distribution in hydroxyapatite crystal lattice. In this work, the protein was also shown to form a tight globular structure comprising three $\alpha$-helices and a short extended strand.

In this report, we present the first X-ray crystal structure from a non-mammalian osteocalcin and the identification of a fourth Gla residue in fish osteocalcin, never before detected in any other osteocalcin molecule, by either X-ray diffraction, NMR, or amino acid sequence analysis. Similarities with the previously published mammalian structures include the confirmation of osteocalcins being rather globular proteins and presenting a patch of negatively charged residues in the same face of the molecule. This feature should be responsible for the high affinity of this protein, in mammals as in fish, for bone mineral content through clustering of the calcium binding sites.

\section{MATERIALS AND METHODS}

Extraction and Purification of Osteocalcin from Fish Bony Tissues. Fish A. regius vertebra and jaw obtained from five specimens ( $\sim 4 \mathrm{~kg}$ wet weight per fish) were cleaned from adhering soft tissues, and the extraction of the Gla-containing proteins was performed on the basis of previously described procedures $(4,5,31)$. Briefly, the mineralized material was ground in a mortar with liquid nitrogen to less than $1 \mathrm{~mm}$ in diameter, extensively washed with $6 \mathrm{M}$ guanidine- $\mathrm{HCl}$ and water, and dried with acetone; $63 \mathrm{~g}$ of clean and dry bone powder was demineralized with $10 \%$ formic acid, dialyzed (SpectraPor 3) against $50 \mathrm{mM} \mathrm{HCl}$, freeze-dried, resuspended in $6 \mathrm{M}$ guanidine- $\mathrm{HCl}, 0.1 \mathrm{M}$ Tris- $\mathrm{HCl}(\mathrm{pH} 9)$, and $10 \mathrm{mM}$ EDTA, and further dialyzed (SpectraPor 3; Spectrum, Gardena, CA) against $5 \mathrm{mM}$ ammonium bicarbonate.

The resulting soluble desalted protein extract, obtained after $5 \mathrm{mM}$ ammonium bicarbonate dialysis, was separated in $5 \mathrm{~mL}$ aliquots and size fractionated at $4{ }^{\circ} \mathrm{C}$ over a $2 \mathrm{~cm}$ $\times 160 \mathrm{~cm}$ Sephacryl S-100 HR (Amersham-Pharmacia) equilibrated with the same buffer. The fractions containing osteocalcin were identified by SDS-PAGE analysis, pooled, and further purified by ion exchange chromatography in a 4 $\mathrm{cm} \times 20 \mathrm{~cm}$ DEAE-A25 Sephadex column (AmershamPharmacia) with a continuous gradient of $0.1 \mathrm{M}$ Tris-HCl $(\mathrm{pH} 8)$ and $0.75 \mathrm{M} \mathrm{NaCl}$. The peak fractions containing osteocalcin were desalted by dialysis (SpectraPor 3) over 2 days against $5 \mathrm{mM}$ ammonium bicarbonate and $10 \mathrm{mM}$ EDTA ( $\mathrm{pH} 8$ ) and further dialyzed against $5 \mathrm{mM}$ ammonium bicarbonate. The purity of the osteocalcin samples was analyzed by SDS-PAGE, and the protein was freeze-dried and stored at $-20{ }^{\circ} \mathrm{C}$.

Protein Crystallization, Diffraction Data Collection, Structure Determination, and Refinement. Crystals of fish A. regius osteocalcin were obtained by the vapor diffusion method using sitting drops in VDX Plates (Hampton Research). Drops of $1.5 \mu \mathrm{L}$ of protein solution, $10 \mathrm{mg} / \mathrm{mL}$ osteocalcin, $5 \mathrm{mM} \mathrm{CaCl}_{2}$, and $30 \mathrm{mM}$ Tris- $\mathrm{HCl}(\mathrm{pH} 8)$, in addition to $1.5 \mu \mathrm{L}$ of well solution, $0.2 \mathrm{M} \mathrm{MgCl}_{2}, 30 \%$ PEG $4 \mathrm{~K}$, and $0.1 \mathrm{M}$ Tris- $\mathrm{HCl}(\mathrm{pH} 8.5)$, were equilibrated against $500 \mathrm{~mL}$ of well solution at $21{ }^{\circ} \mathrm{C}$ over the course of $2-3$ weeks. Heavy atom crystal derivatives were tentatively produced by soaking osteocalcin crystals in drops of $5 \mathrm{mM}$ metal salt (salts of $\mathrm{Au}, \mathrm{Br}, \mathrm{I}, \mathrm{Pb}, \mathrm{Pt}$, and $\mathrm{Sm}$ were tried), 0.1 $\mathrm{M} \mathrm{MgCl}_{2}, 0.1 \mathrm{M}$ Tris- $\mathrm{HCl}$ (pH 8.5), 10\% PEG 4K, and 25\% glycerol. This solution without the heavy metal was used to wash out excess metal within crystal solvent channels and was necessary for stabilizing the crystals for X-ray cryo measurements.

Native and several putative heavy atom derivative diffraction data sets were collected in-house and at ESRF (Grenoble, France). The highest-resolution diffraction data were obtained from a crystal measured at station ID14-EH1 (ESRF) that had been tentatively soaked in a $\mathrm{Sm}^{2+}$ solution; however, neither produced a significant anomalous signal, nor was any heavy atom site found in the structure. These data were therefore used as native for structure solution and refinement purposes. They resulted from the merging of three data sets measured at different crystal to detector distances, corresponding to low-, medium-, and high-resolution passes, interleaved by crystal translations so that in each pass the beam illuminated fresh crystal regions.

Diffraction intensities (Table 1) were examined with XdisplayF, processed with DENZO, and scaled with SCALEPACK from the HKL suite (32). Using the HKL2MAP graphics user interface (T. Pape and T. Schneider, personal communication), native and putative derivative data sets were scaled together and analyzed with SHELXC (G. M. Sheld- 


\begin{tabular}{|c|c|c|}
\hline $\begin{array}{l}\text { crystal dimensions }\left(\mathrm{mm}^{3}\right) \\
\text { crystal form } \\
\text { space group } \\
\text { cell dimensions }(\AA) \\
\text { no. of molecules per au } \\
\text { solvent content }(\%)\end{array}$ & \multicolumn{2}{|c|}{$\begin{array}{l}1 \times 0.16 \times 0.14 \\
\text { colorless prisms } \\
P 3_{2} 21 \\
a=b=48.70, c=119.1 \\
3 \\
54.2\end{array}$} \\
\hline & native & Pt derivative \\
\hline synchrotron station & $\begin{array}{l}\text { ESRF, } \\
\text { ID14-EH1 }\end{array}$ & $\begin{array}{l}\text { ESRF, } \\
\quad \text { ID14-EH1 }\end{array}$ \\
\hline wavelength $(\AA)$ & 0.934 & 0.934 \\
\hline resolution limits $(\AA)$ (outer shell) & $\begin{array}{l}39.75-1.40 \\
\quad(1.45-1.40)\end{array}$ & $\begin{array}{l}42.23-2.00 \\
\quad(2.07-2.00\end{array}$ \\
\hline global complet & $98.5(96.1)$ & $99.2(93.2)$ \\
\hline no. of measured intensities & 719298 & $156588^{a}$ \\
\hline no. of unique intensities & 32430 & $21465^{a}$ \\
\hline & 22.2 & \\
\hline$R_{\text {merge }}(\%)$ (outer shell) & $5.6(30.9)$ & $5.4(8.5)^{a}$ \\
\hline
\end{tabular}

${ }^{a}$ Friedel mates considered as independent measurements.

rick, personal communication), the heavy atom substructure was determined with SHELXD (33) and the phase problem solved with SHELXE (34). ARPwARP (35), as implemented in CCP4i $(36,37)$, was used to automatically trace the three polypeptide chains in the experimental maps. The structure was anisotropically refined with REFMAC (38) and SHELXL (39), using a thin shells $R_{\text {free }}$ set of 893 reflections $(2.75 \%$ of the total) defined by SHELXPRO (39). The fit of the molecular model against sigmaA $2 m F_{\mathrm{o}}-D F_{\mathrm{c}}$ electron density maps (40) was checked and optimized on a graphics workstation using XFIT from the XtalView package (41).

The structure was determined by the single isomorphous replacement with anomalous scattering method (SIRAS), using a crystal that had been soaked in a potassium tetrachloroplatinate solution, the only salt solution, among all others that were tested, that delivered a clear heavy atom substructure. The heavy atom substructure comprised four sites with relative occupancies of 1.0, 0.8, 0.7, and 0.5 arbitrary unit (33). The maps obtained with the electron density modification program SHELXE (34) clearly showed polypeptide motifs (Figure 1) with well-defined side chains where amino acids could be recognized (e.g., Phe, Tyr, and His side chain rings with respective holes). The automatically obtained structural models were checked against sigmaA Fourier maps and completed on a graphics workstation, alternating with atomic anisotropic refinement using REFMAC (38) until the convergence of reliability factors ( $R_{\text {free }}=24.1 \%$ and $R=21.5 \%$ ). The occupancy refinement of side chains with multiple conformers was further improved with SHELXL (39) leading to the following: $R_{\text {free }}=23.0 \%$ and $R=18.8 \%$.

Mass Spectrometry Analysis. Chromatography was performed on $10 \mu \mathrm{g}$ of osteocalcin using a $1.0 \mathrm{~mm} \times 15 \mathrm{~mm}$ Vydac $\mathrm{C} 4$ reserve phase HPLC column and separated using a gradient of $0.05 \%$ TFA in $2 \%$ acetonitrile and $0.0475 \%$ TFA in $90 \%$ acetonitrile. Fractions were analyzed using an Applied Biosystems Qstar Hybrid quadrupole TOF mass spectrometer. The approximate percent of the three- and fourGla species of osteocalcin was calculated on the basis of the relative intensities of the $[\mathrm{M}+3 \mathrm{H}]^{3+}$ ions. These percentages correlated with the relative UV absorbance of the two peaks obtained in the HPLC chromatogram of osteocalcin.

\section{RESULTS}

Structure Content and Model Validation. The quality of the osteocalcin models to $1.4 \AA$ resolution was assessed with PROCHECK (43), revealing that the overall stereochemistry is similar or better than that of the set of reference proteins of PROCHECK at the same resolution, with only one amino acid localized in the additional allowed and none in the disallowed regions of the Ramachandran diagram (other refinement statistics in Table 2).

The asymmetric unit contains three crystallographically independent osteocalcin molecules, termed A-C (Figure 2), in a total of 126 amino acid residues (the first two to four $\mathrm{N}$-terminal residues are missing, because they are not visible in the electron density maps). Globally, 17 residues were modeled with alternating side chains, and five residues have their side chains not totally inside $1 \sigma\left(0.07 \mathrm{e}^{\mathrm{A}} \mathrm{A}^{3}\right)$ electron density maps. In addition to the protein moiety and the 191 solvent molecules that could be satisfactorily refined as waters, the electron density maps also show a set of blobs within bonding distances of protein carboxylic groups and other water molecules. As the crystallization conditions

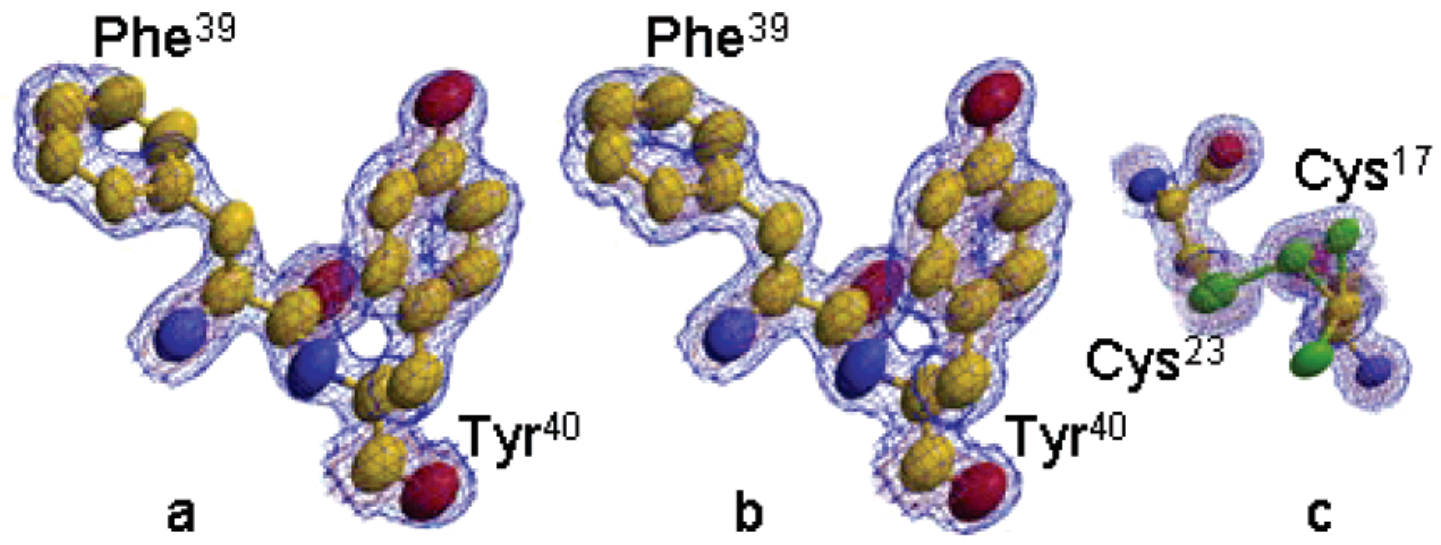

FIGURE 1: Electron density maps at $1 \sigma$ and $3 \sigma$ (blue and pink, respectively) and refined model in stick and anisotropic ellipsoids at $20 \%$ probability. (a) Experimental SHELXE (34) SIRAS map around residues Phe ${ }^{39}$ and $\mathrm{Tyr}^{40}$ of molecule B. (b) Final refined SHELXL (39) sigmaA $2 m F_{\mathrm{o}}-D F_{\mathrm{c}}$ map (40) around the same residues. (c) Final refined SHELXL sigmaA $2 m F_{\mathrm{o}}-D F_{\mathrm{c}}$ map around residues Cys ${ }^{17}$ and $\mathrm{Cys}^{23}$ of molecule B, showing their partially broken thioether bridge, with two alternating positions for the SG atom in Cys ${ }^{23}$, and three positions of the $\mathrm{SG}$ atom in $\mathrm{Cys}^{17}$, including $\chi_{1}$ conformations trans and gauche(-). This figure was produced using XFIT (41) and RASTER3D (42). 


\begin{tabular}{ll}
\hline Table 2: Refinement and Model Statistics & \\
\hline$R_{\text {free }}(\%)$ & 23.0 \\
$R_{\text {work }}(\%)$ & 18.8 \\
no. of amino acid residues & 126 \\
no. of discrete alternating side chains & 17 \\
no. of whole residues not visible at $1 \sigma$ & 4 \\
no. of other side chains not visible at $1 \sigma$ & 5 \\
no. of protein atoms & 890 \\
no. of fully occupied Mg ions & 7 \\
no. of partially occupied Mg ions & 1 \\
no. of solvent waters & 191 \\
Wilson $B\left(\AA^{2}\right)$ & 20 \\
average $B$ for buried atoms $\left(\AA^{2}\right)$ & 24 \\
rms deviations from target values $(44)$ & \\
$\quad$ bond lengths $(\AA)$ & 0.011 \\
angle distances $(\AA)$ & 0.030 \\
\hline
\end{tabular}

involved a 40-fold relative excess of $\mathrm{Mg}^{2+}$ over $\mathrm{Ca}^{2+}$, those positions were assigned as seven fully occupied $\mathrm{Mg}^{2+}$ cations, an assumption confirmed by their 6-fold coordination, refined atomic displacement parameters (average of 20.9 $\AA^{2}$ ), and bond distances. Examination of difference Fourier maps suggested an eighth $\mathrm{Mg}^{2+}$ site, which refined to a partial occupancy of 0.2 . The metal-oxygen coordination involves a total of $27 \mathrm{Mg}-\mathrm{O}$ bonds to carboxylic groups with an average distance of 2.05(7) $\AA$, in the range of 1.75$2.15 \AA$, and in the case of the 21 waters completing the $\mathrm{Mg}$ coordination an average distance of 2.12(9) $\AA$, in the range of 1.84-2.35 $\AA$. These values are equivalent to those obtained from high-resolution structures: 2.11(9) $\AA$ (average) in the range of 2.03-2.34 $\AA$ and 2.07(3) $\AA$ (average) in the range of $2.00-2.15 \AA$, respectively (46). It should also be noted that although the refinement included standard protein stereochemical restraints (44), the metal-donor distances reported in this work were refined without any target value.

$\mathrm{Mg}$ ions are smaller than $\mathrm{Ca}$ ions and therefore impose a tighter coordination: the metal-oxygen distances around $\mathrm{Mg}$ are ca. $0.3 \AA$ shorter than around $\mathrm{Ca}$ (46), and the octahedral geometry of oxygen ligands around $\mathrm{Mg}$ deviates on average $1.7^{\circ}$ from ideal values, while $\mathrm{Ca}$ has a much looser coordination geometry with an average deviation of $5.4^{\circ}$ from ideality (47). As the $\mathrm{Mg}$ ions in our structure are either partially facing the solvent or intercalated between two osteocalcin molecules, it is feasible to envisage that in its natural physiological environment these positions can be occupied by the slightly bulkier $\mathrm{Ca}$ ions with a similar, eventually looser, geometric arrangement.

The N-terminal region is always visible from Leu ${ }^{5}$ upward, because its side chain folds into the interior part of an elbow built by the third helix and the C-terminal region. Molecule A could be easily modeled from Lys ${ }^{3}$ upward, in contrast to molecule $\mathrm{B}$ or $\mathrm{C}$ that was modeled beginning at $\mathrm{Glu}^{4}$ or $\mathrm{Leu}^{5}$, respectively, because in molecule $\mathrm{A}$ the conformational space of $\mathrm{Lys}^{3}$ and $\mathrm{Glu}^{4}$ is confined, due to packing contacts with $\mathrm{Asn}^{20}, \mathrm{Met}^{21}$, and $\mathrm{Gla}^{25}$ of a neighboring molecule C. In the three molecules, the first two alanines of the $\mathrm{N}$-terminus were not visible in the electron density maps, probably due to disordered conformations in the surrounding solvent. In contrast, the $\mathrm{C}$-terminal region is always well defined as the large side chain of $\mathrm{Phe}^{45}$ may function as a hydrophobic "anchor" and is preceded by chain-rigidifying prolines in positions 42 and 44 .

The three refined molecules show a partially broken disulfide bridge $(\mathrm{S}-\mathrm{S})$ between $\mathrm{Cys}^{17}$ and $\mathrm{Cys}^{23}$, with the

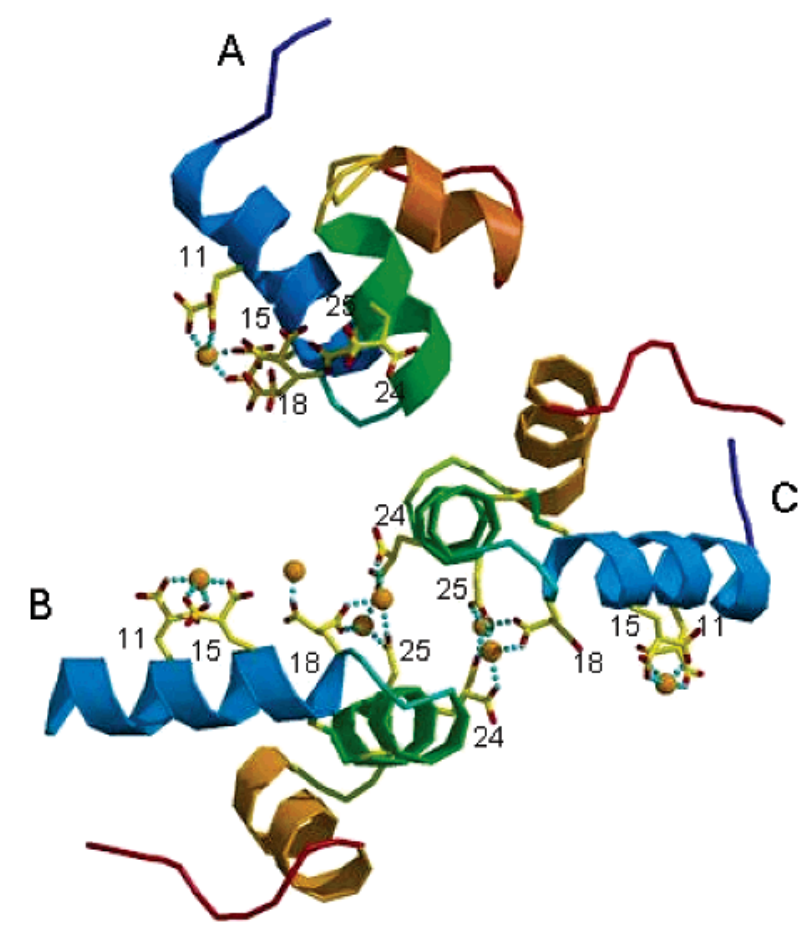

FIGURE 2: Cartoon of the three A. regius osteocalcin molecules in their relative positions in the asymmetric unit. Molecules $\mathrm{A}-\mathrm{C}$ are represented with their $\mathrm{N}$-terminus to $\mathrm{C}$-terminus colored from blue to red, respectively; the side chains of Gla residues $11,15,18$, and 25 and of $\mathrm{Asp}^{24}$ are shown as sticks, and attached $\mathrm{Mg}^{2+}$ cations are represented as orange spheres with metal coordinations as dotted blue lines. The side chains of Gla residues and $\mathrm{Asp}^{24}$ residues in helices $\alpha 1$ and $\alpha 2$ are localized on an external surface region of the molecule where metal binding occurs, while helix $\alpha 3$ and the $\mathrm{N}$ - and C-terminal regions project away from this area. The first two Gla residues (11 and 15) coordinate one $\mathrm{Mg}^{2+}$ ion in each of the molecules. Molecules B and C are related by a local 2-fold rotation axis, approximately perpendicular to the plane of the paper. Their Gla ${ }^{18}, \mathrm{Asp}^{24}$, and $\mathrm{Gla}^{25}$ residues share the coordination of two pairs of $\mathrm{Mg}^{2+}$ ions. In molecule $\mathrm{A}$, these residues face the solvent without any visible metal bound. $\mathrm{Gla}^{18}$ of molecule B further coordinates a partially occupied cation. Water molecules (not represented) complete in all cases for a 6-fold $\mathrm{Mg}$ coordination. This figure was produced using MOLSCRIPT (45) and RASTER3D (42).

SG atom of this residue refined in two alternating positions and the SG atom of $\mathrm{Cys}^{17}$ refined up to three positions (Figure 1c). Both alternating positions of the SG atom of Cys $^{23}$ correspond to dihedral $\chi_{1}$ in the trans conformation, one in bonding position (occupancies of 0.70, 0.56, and 0.54 for molecules $\mathrm{A}-\mathrm{C}$, respectively) and the other as nonbonded and pulled away from the $\mathrm{S}-\mathrm{S}$ bond (occupancies of 0.30 , 0.44 , and 0.46 , respectively). Cys ${ }^{17}$ also has the $\mathrm{SG}$ atom in $\mathrm{S}-\mathrm{S}$ bonding position with $\chi_{1}$ in the trans conformation (occupancies of $0.70,0.56$, and 0.54 , respectively), but shows up to two different unbonded positions, with either $\chi_{1}$ still in the trans conformation but pulled away (occupancies of $0.30,0.28$, and 0.25 , respectively) or with $\chi_{1}$ in the gauche (-) conformation (occupancies of 0.16 and 0.21 in molecules B and C, respectively). The doubled nonbonded conformations of $\mathrm{Cys}^{17}$ are only apparent for molecules B and $\mathrm{C}$, where the percentage of broken $\mathrm{S}-\mathrm{S}$ bonds is close to $50 \%$.

To assess if the cleavage of the disulfide bond was a consequence of the crystal irradiation by X-rays, a known feature in synchrotron data (48), another refinement was 
performed using only the initial $60^{\circ}$ of diffraction data, corresponding to a completeness of $98 \%$ and a redundancy of 6.4. Such an initial data set should be less affected by radiation damage. Refinement with this data subset led to $R$ factors that were $\sim 1 \%$ higher than for the refinement using all data $\left(R_{\text {free }} 0^{60^{\circ}}=24.0 \%\right.$ and $\left.R^{60^{\circ}}=20.0 \%\right)$. The obtained bonded and unbonded occupancies of SG atoms were $\sim 0.74$ and $\sim 0.26$, respectively, both with $\chi_{1}$ in the trans conformation. The higher bonded/unbonded ratio in the refinement using only initial data confirmed that crystal exposure to $\mathrm{X}$-rays induced further $\mathrm{S}-\mathrm{S}$ splitting and the appearance of the gauche(-) conformation in molecules B and C, although it did not lead to a conclusive exclusion of the unbonded Cys-Cys bridge in the native protein. Another difference between the two refinements concerns the partial occupancy of a metal site. The refinement using only the first $60^{\circ}$ of data led to a partial occupancy of 0.56 , almost 3 times higher than that using all the diffraction data, indicating that this metal coordination tends to disappear with the X-ray irradiation. The final model is an average of the structure in the space of the crystal and during the time of irradiation with $\mathrm{X}$-rays, a structure that changes during the $\mathrm{X}$-ray experiment due to X-ray damage. The final $R$ factors reflect such a model inaccuracy, and although they show values in usually accepted ranges, they are not as low as those found in other structures at similar resolution. However, the difference and ratio between $R$ and $R_{\text {free }}$ (3.8\% and 1.2, respectively), considered more reliable validation parameters, fall well within acceptable ranges (49).

Overall Structure Description. A. regius osteocalcin is an $\alpha$-helix protein (ca. 70\% $\alpha$-helix), comprising three $\alpha$-helices that pack tightly together, with the first and second helices arranged in a V-shaped form and the third helix nestling between them (Figure 2). Helix $\alpha 1$ involves residues $6-19$, helix $\alpha 2$ residues $20-31$, and helix $\alpha 3$ residues $31-41$. The remaining residues, at the $\mathrm{N}$ - and $\mathrm{C}$-terminal regions, show no secondary structure characteristics, with the last five C-terminal essentially hydrophobic residues forming an extended chain running almost antiparallel to helix $\alpha 1$.

Interhelix contacts are mainly hydrophobic in nature (hydrogen bonds are found mainly within each helix) in addition to a disulfide bridge, between conserved $\mathrm{Cys}^{17}$ and $\mathrm{Cys}^{23}$ residues, which connects the end of helix $\alpha 1$ to the beginning of helix $\alpha 2$. Osteocalcin is largely formed by noncharged residues, of which the majority are apolar (62\%) with their side chains folded into the molecule hydrophobic core, while the remaining polar and charged residues are localized on the surface. Such a distribution together with the compact helical arrangement makes osteocalcin a rather globular protein despite its relatively small size.

The three molecules are very similar to each other (Table 3 ) with molecule A deviating slightly from the other two. The few differences are located in the terminal regions, at helical ends ( $\mathrm{Thr}^{19}$ and $\mathrm{Ala}^{29}$ ), or at side chains involved in packing interactions. Molecule A has average atomic displacement parameters of $36.1 \AA^{2}$, significantly higher than the corresponding values in molecules $\mathrm{B}$ and $\mathrm{C}$ (23.4 and $22.2 \AA^{2}$, respectively). This main difference between the three molecules reflects their different number of packing contacts: while $\mathrm{A}-\mathrm{B}$ and $\mathrm{A}-\mathrm{C}$ contacts number 18 and 48 to $3.8 \AA$, respectively, $\mathrm{B}-\mathrm{C}$ contacts number 72 . This shows that molecule $\mathrm{A}$ has a less constrained environment in the

\begin{tabular}{lccccc}
\hline \multicolumn{7}{l}{ Table 3: } & Superposition of Osteocalcins ${ }^{a}$ \\
\hline & arg_A & arg_B & arg_C & porc & bovi \\
\hline arg_A & 43 & 0.6 & 0.6 & 0.8 & 1.5 \\
arg_B & 100 & 42 & 0.3 & 0.9 & 1.5 \\
arg_C & 100 & 100 & 41 & 1.0 & 1.7 \\
porc & 49 & 49 & 49 & 37 & 1.6 \\
bovi & 32 & 32 & 32 & 44 & 34 \\
\hline
\end{tabular}

${ }^{a}$ Least-squares multibody fit of CA positions (to a cutoff of $3.5 \AA$ ) from available osteocalcin 3D models: arg_A, arg_B, and arg_C refer to $A$. regius molecules $\mathrm{A}-\mathrm{C}$, respectively; porc refers to the porcine model (30), and bovi refers to the best representative conformer from the bovine ensemble of structures (with a rmsd of $1.09 \AA$ ) (26). From the top left to the bottom right: number of residues. From the top right to diagonal: $\mathrm{C} \alpha$ rmsd values $(\AA)$. Diagonal to the bottom left: overlapping levels of sequence identity $(\%)$.

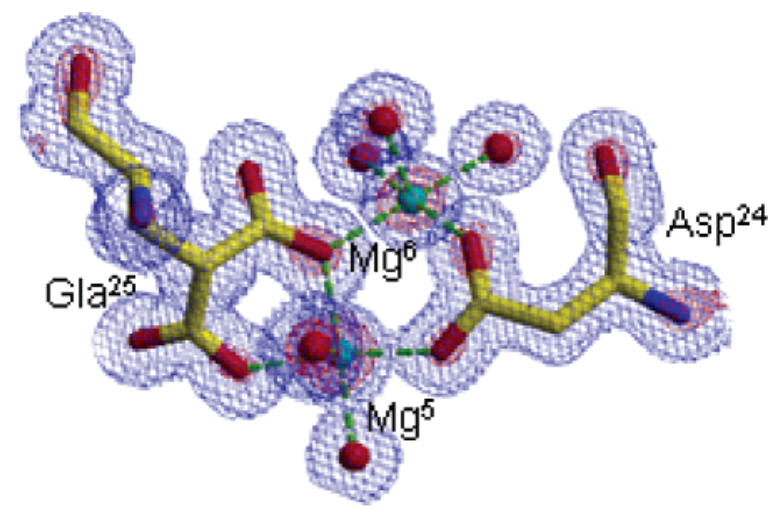

FIGURE 3: $\gamma$-Carboxylation of the glutamic acid residue at position 25 . Detail of the electron density map at $1 \sigma$ (blue) and $5 \sigma$ (red), around residues (stick) $\mathrm{Asp}^{24}$ and $\mathrm{Gla}^{25}$ of molecules $\mathrm{C}$ and $\mathrm{B}$, respectively, which coordinate two magnesium ions (cyan spheres) together with water molecules (red spheres) and residue $\mathrm{Gla}^{25}$ of molecule B (not shown).

crystal than the other two molecules that otherwise are interacting closely with each other.

Identification of Modified Glutamic Acids (Gla residues). A. regius osteocalcin contains five Glu residues, and our crystal structure of the native protein shows clearly their side chains. In contrast with previously studied osteocalcins, four of the five Glu residues (residues 11, 15, 18, and 25) in the A. regius osteocalcin are modified to Gla residues, while all other osteocalcins studied to date have only three Gla residues. The extra Gla site was unambiguously identified in the electron density maps (Figure 3) for the three independent molecules of the structure. Those four doubly charged residues together with $\mathrm{Asp}^{24}$ and $\mathrm{Asp}^{28}$ are concentrated in a common surface region, located on the same side of the molecular surface, as depicted in Figure 4. This suggests that the high affinity of osteocalcin for bone mineral may derive from the clustering of calcium binding sites on this face of the protein.

To cross validate the $\mathrm{X}$-ray analysis showing a fourth Gla residue not previously described in other species, a LCMS analysis of $A$. regius osteocalcin was performed and showed that the bulk of the sample is full-length osteocalcin (residues 1-45), with four Gla (79.1\%) or three Gla (20.5\%) residues, with no evidence of noncarboxylated osteocalcin. The crystallographic analysis is not able to resolve the distribution of $\gamma$-carboxylation among the available Glu residues, as the final models are the averaged structures throughout the entire crystal contents. 


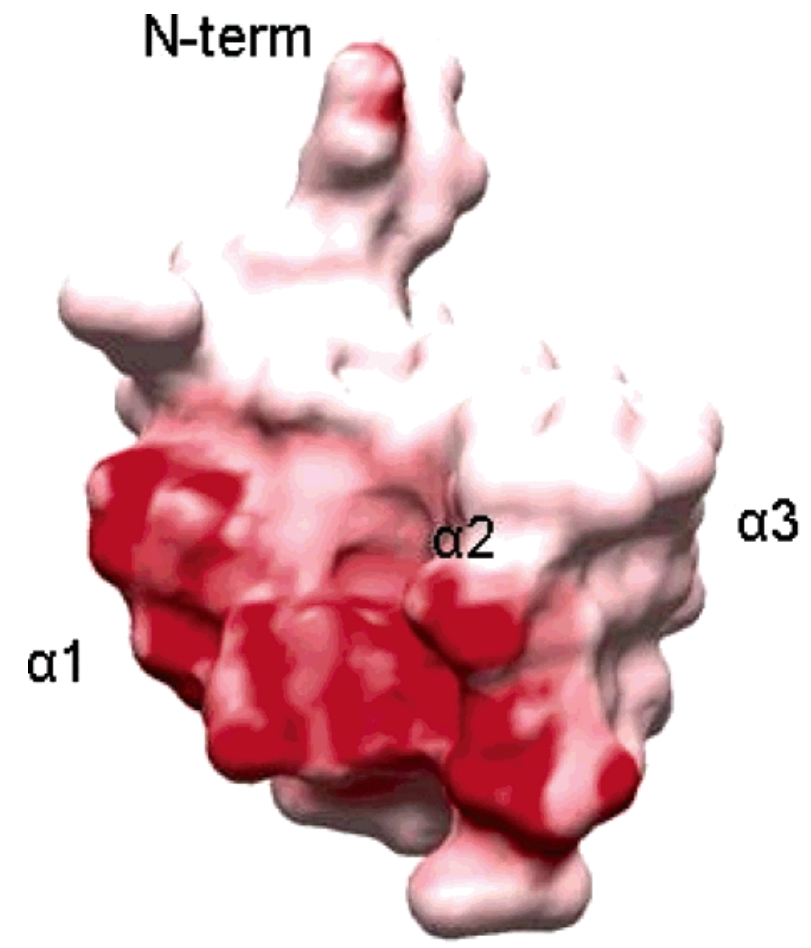

FIgURE 4: Surface electrostatic distribution on osteocalcin. Representation of the charge distribution on the surface of molecule A, with an orientation similar to that of Figure 2. The concentration of acidic residues near the convergence of helices $\alpha 1$ and $\alpha 2$ produces a localized, highly negative surface region (in red) suited for coupling with cations. This suggests that in bone the protein may associate with calcium atoms on the bone mineral surface. This figure was produced using GRASP (50).

\section{DISCUSSION}

Molecules B and C are related by a noncrystallographic 2-fold axis, have the largest number of contacts among packing neighbors, and share the coordination of four $\mathrm{Mg}^{2+}$ ions from the crystallization buffer. The arrangement of osteocalcin molecules in pairs sharing coordinated metals is also described in the porcine structure (30). However, a comparison of the interactions between molecules B and C and those in the porcine structure reveals that these "dimers" bear only residual similarities. In the porcine structure, a crystallographic 2-fold axis relates two neighboring copies of the unique molecule. Their acidic surface regions, involving helices $\alpha 1$ and $\alpha 2$, face each other with the entire content of cations interleaving them. In A. regius, the two pairs of shared $\mathrm{Mg}^{2+}$ ions are localized between a part of the negatively localized charged region, involving only helix $\alpha 2$, with the remaining metal sites being coordinated by each of the molecules alone. Furthermore, our structure from $A$. regius shows an asymmetric unit that also contains molecule A, which has the negatively charged region surrounded only by solvent with a single localized metal site, visible in the electron density. These crystallographic dimers seem therefore to be mainly artifacts of the crystal packing.

Although $\gamma$-carboxylation of three Glu residues is a welldocumented characteristic among osteocalcins, the observation of the fourth carboxylation at $\mathrm{Glu}^{25}$ in A. regius is, to our knowledge, an as yet undescribed feature in any of the other characterized osteocalcins, in particular for the other two three-dimensional (3D) models present in the Protein

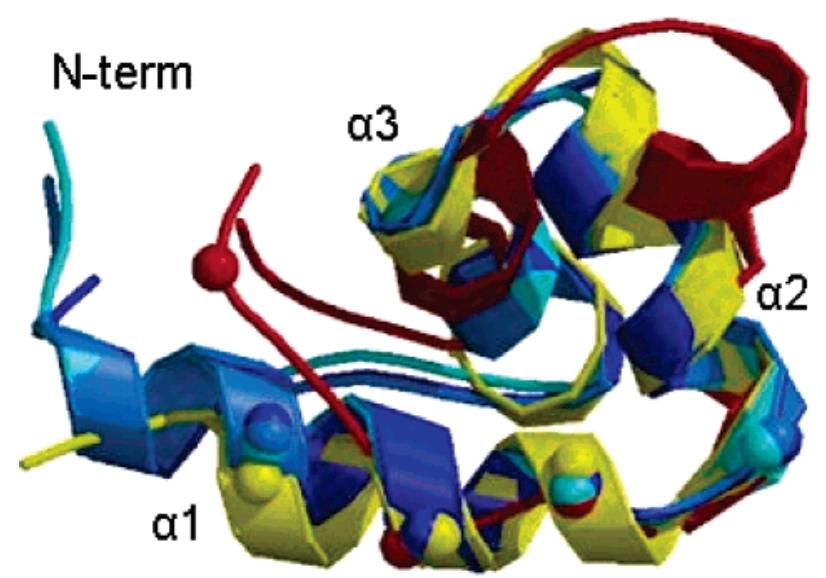

FIGURE 5: Cartoon representation of the superposition of osteocalcins. A. regius molecules A (cyan), B (medium blue), and C (dark blue), with $\gamma$-carboxyglutamic acid $\mathrm{C} \alpha$ positions represented as spheres, superpose clearly with the porcine structure (yellow) (30), while the bovine structure (red) (26) shows much less secondary structure and a poor match of Gla residues. This figure was produced using MOLSCRIPT (45) and RASTER3D (42).

Data Bank. Glu ${ }^{25}$ is a solvent accessible, highly conserved residue, localized in the central turn of helix $\alpha 2$. It is in general preceded by an acidic residue in position 24 (Asp or Glu) and is followed by a hydrophobic residue (Met or Leu) in position 26 (Figure 6). Those osteocalcins are both mammalian in origin, a 2D ${ }^{1} \mathrm{H}$ NMR study of bovine osteocalcin (26) and an X-ray model of porcine osteocalcin (30). The superposition of the osteocalcin models using MODELLER (51) (Table 3 and Figure 5) shows that while the X-ray structures have rather similar secondary structure topologies, the bovine model has a much lower level of sequence identity and has almost no secondary structure and the 3D distribution of their Gla residues bears no similarity with that of the other two osteocalcins.

The 3D fit of osteocalcin models (Table 3 and Figure 5) shows that they consistently superpose the $\mathrm{C} \alpha$ atoms of their metal chelating residues, Gla and Asp, in particular, for the two X-ray structures. The spatial distribution of these residues has been recognized to be consistent with the interatomic distances of calcium sites in hydroxyapatite crystals $(15,26$, 30 ), confirming the proposed biological role for osteocalcin as a controlling agent in bone.

Molecule A of our structure only shows one $\mathrm{Mg}^{2+}$ coordination (using Gla residues 11 and 15), with its $\mathrm{Gla}^{25}$ side chain well defined in the electron density but with $\mathrm{Gla}^{18}$ disordered in the solvent. The existence of a Gla residue with a flexible side chain together with the different way in which molecule A compared with molecules B and C shares metal coordination suggests a possible adaptability of osteocalcin molecules for chelating variable calcium distributions.

A BLAST (52) search of protein sequences using the $A$. regius sequence as a template retrieved a group of five highly homologous fish (Lepomis macrochirus, Tetraodon nigroviridis, Sparus aurata, Fugu rubripes, and Xiphias gladius) osteocalcins, with $E$ scoring values (representating the number of hits one can "expect" to find by chance for a given database; the lower the $E$ value, the more significant the score) of less than $2 \mathrm{e}-10$, followed by other osteocalcins, including those of mammalian origin, with scoring values ranging from $1 \mathrm{e}-4$ to 8 . Multiple-sequence alignment 


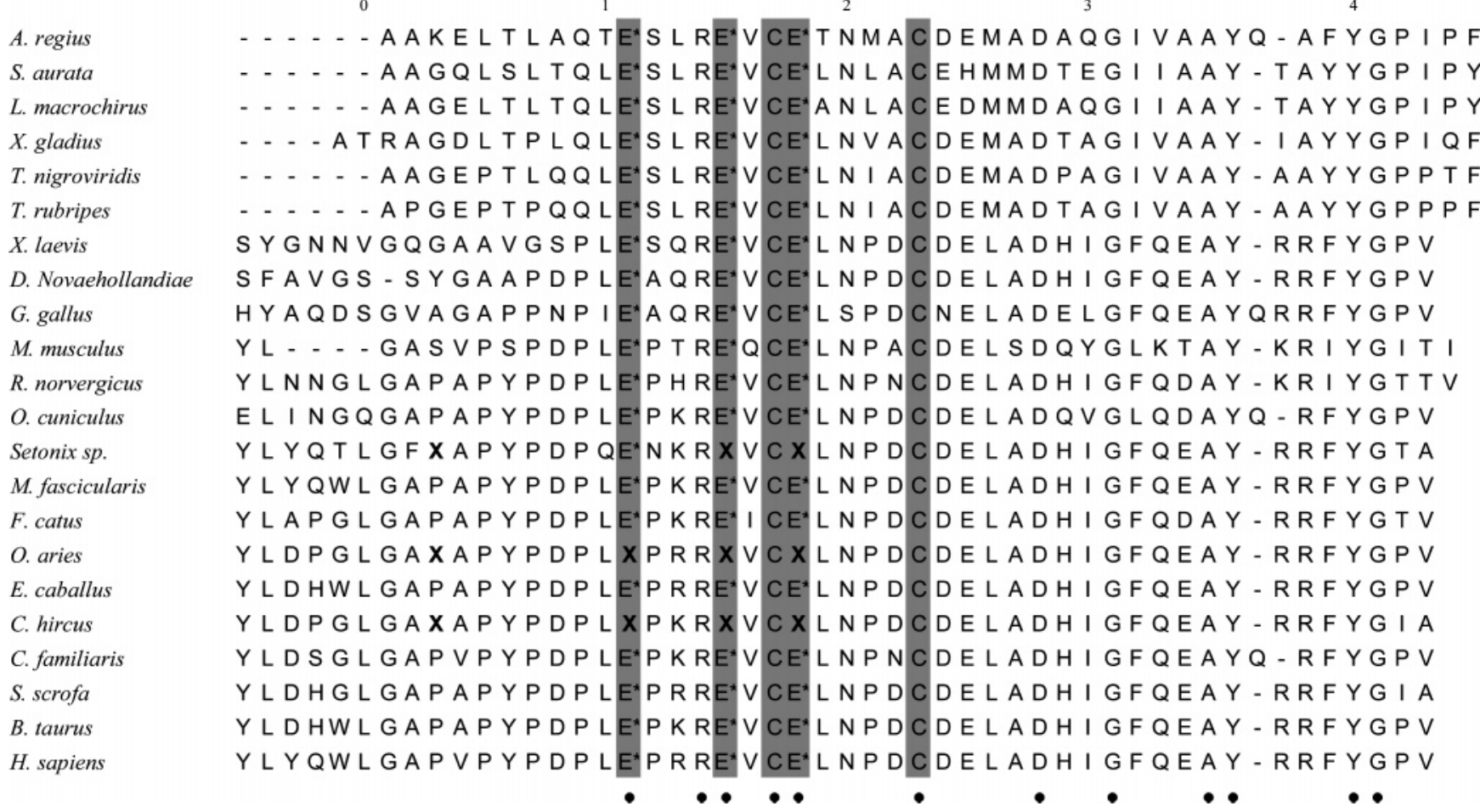

FIGURE 6: Amino acid sequences of all known mature osteocalcins. The sequences are aligned to give maximal homology. Residues are numbered according to residue 1 of mature A. regius osteocalcin. $\gamma$-Carboxyglutamate residues are denoted with $\mathrm{E}^{*}$. Dashes indicate gaps in the sequence introduced to increase the level of homology. The conserved residues in the EXXXEXCEXXXXC motif are highlighted in gray. $\mathrm{X}$ in bold corresponds to an undetermined amino acid residue from $\mathrm{N}$-terminal sequence data. Identical residues are marked with a dot. The GenBank accession numbers are AF459030 for A. regius, AF048703 for S. aurata (seabream), A42794 for L. macrochirus (bluegill), P02823 for X. gladius (swordfish), AF055576 for Xenopus laevis, P15504 for emu (Dromaius novaehollandiae), U10578 for chicken (Gallus gallus), NM_007541 for mouse (Mus musculus), X04141 for rat (Rattus norvegicus), P39056 for rabbit (Oryctolagus cuniculus), 1005180C for wallaby (Setonix sp.), P0283005 for monkey (Macaca fascilularis), P02821 for cat (Felis catus), 1010264A for sheep (Ovis aries), P83005 for horse (Equus caballus), 1005180A for goat (Capra hircus), AAB27444 and P81455 for dog (Canis familiaris), 1005180B for pig (Sus scrofa), X53699 for bovine (Bos taurus), and X53698 for human (Homo sapiens). The sequences of freshwater puffer (Tetraodon nigroviridis) and fugu (Takifugu rubripes) were obtained from ref 53.

provided evidence for a homologous region among osteocalcins, residues 11-41 in A. regius numbering (Figure 6), that includes position 25 and its neighbors, and suggested that the osteocalcin in these fish species also contains four Gla residues, a possibility that will require further investigation. Mammalian osteocalcins also have a Glu residue which corresponds to $\mathrm{Glu}^{25}$ in A. regius, yet no Gla has been identified in the mammalian proteins at this position. The intrinsically lower precision of the $2 \mathrm{D}{ }^{1} \mathrm{H}$ NMR structure for bovine osteocalcin (26) and the relaxed refinement statistics of the X-ray study of porcine osteocalcin (30) do not preclude entirely the possibility of a further $\gamma$-carboxylation in those mammalian structures. However, this is very unlikely, at least for the bovine and human proteins, given the fact that they were sequenced using the generation of peptides, and amino acid analysis was carried out to support the sequence data, which implies that any further $\gamma$-carboxylation would have been detected. It is therefore intriguing to wonder why $\mathrm{Glu}^{25}$ is modified in A. regius but has not been seen in the mammalian structures, although it is also possible that this arises from differences in their particular posttranslational modification catalyzed by the vitamin $\mathrm{K}$ dependent (VKD) $\gamma$-glutamyl carboxylase. It is worth noting that partial carboxylation of glutamic acid has been observed at residue 17 of human osteocalcin (54) and at residue 2 of the matrix Gla protein from a variety of species $(7,55)$. The partial carboxylation of the matrix Gla protein and of human osteocalcin is known to be due to defective post-translational carboxylation prior to secretion, not to decarboxylation of the mature protein after secretion.

Carboxylation of multiple Glu residues is proposed to be accomplished via an integrative mechanism, i.e., affecting all modifications as a result of a single binding event, which occurs with at least some order and involves carboxylation of the enzyme itself (56-58). The carboxylase has a highaffinity binding site for the sequence contained in the propeptide of all VKD proteins, which in the case of matrix Gla proteins is part of the mature form. Mutations in any of the three highly conserved positions (Phe at position -15 , Ala at position -9 , and Leu at position -6 ; Figure 7) have been shown to decrease the affinity of the carboxylase for the coagulation-related VKD proteins (59). The fact that all MGPs have two of the three residues conserved could be related to the fact that they have fewer $\gamma$-carboxylated residues than the coagulation-related VKDs. The same could be argued when all osteocalcins are compared since the only osteocalcin known to have a fourth Gla residue (A. regius) has two of the three conserved residues (Phe at position -15 and Ala at position -9). Since osteocalcin from $S$. aurata also has the same two conserved residues as $A$. regius, it would be interesting to investigate if it also has a fourth Gla residue at position 24 .

It also appears that the carboxylase enzyme has high affinity for the osteocalcin Gla domain. This was suggested 


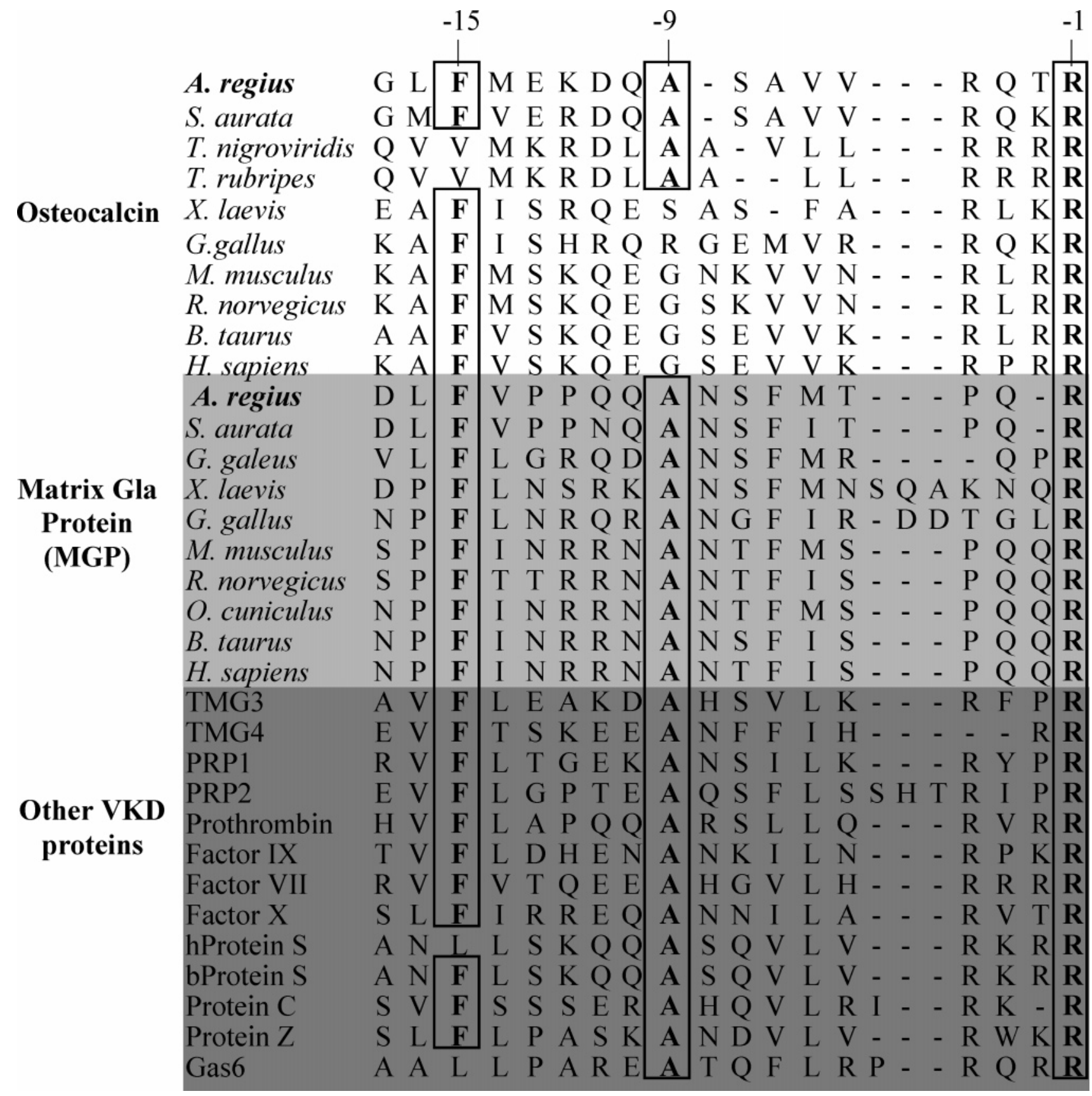

FIGURE 7: Conservation of the putative $\gamma$-carboxylase recognition site sequence in A. regius osteocalcin propeptide and comparison with other available VKD proteins. The amino acids in the propeptide region (except for MGPs that do not have a propeptide, this region being part of the mature protein) of known VKD proteins are aligned to give a maximum degree of homology. Amino acid sequence positions are numbered according to residue -1 of A. regius osteocalcin propeptide sequence, deduced on the basis of comparison between the mature protein and the entire cDNA sequence (5). The conserved residues at positions -1 (Arg), -9 (Ala), and -15 (Phe) are boxed. Osteocalcin sequence GenBank accession numbers are AF459030 for A. regius, AF048703 for seabream ( $S$. aurata), AF055576 for Xe. laevis, U10578 for chicken (G. gallus), NM007541 for mouse (M. musculus) (OG1, osteocalcin), X04141 for rat (R. norvegicus), X53699 for bovine (B. taurus), and X53698 for human (H. sapiens). The sequences of freshwater puffer (T. nigroviridis) and fugu (Ta. rubripes) were obtained from ref 53. MGP sequence GenBank accession numbers are AF334473 for A. regius, AY065652 for seabream (S. aurata), P56620 for shark (G. galeus), AF055588 for X. laevis, Y13903 for chicken (G. gallus), NM012862.1 for rat (R. norvegicus), AF210379 for bovine (B. taurus), NM000900 for human (H. sapiens), NM008597 for mouse (M. musculus), and D21265 for rabbit (O. cuniculus). All other VKD protein sequences presented here are from human unless otherwise stated. The respective sequences were obtained: TMG3 and TMG4 from ref 61, PRP1 and PRP2 from ref 62, prothrombin, factor IX, and factor X from ref 63, factor VII from ref 64, hProtein S (human Protein S) from ref 65, bProtein S (bovine Protein S) from ref 66, Protein C from ref 67, Protein Z from GenBank accession number P22891, and Gas6 from ref 68.

by studies with mammalian osteocalcin carboxylation which support the concept of a Glu-binding site (60). However, to date, nothing is known concerning this type of affinity in fish osteocalcins or the possible reason for the presence of this fourth Gla residue. The elucidation of the structural determinants that contribute to the recognition of osteocalcin and vitamin $\mathrm{K}$ by the carboxylase, still not completely known, should provide the necessary basic information for us to begin exploring the reasons for the presence of this additional residue in A. regius osteocalcin. 


\section{ACKNOWLEDGMENT}

ESRF is thanked for the provision of facilities and technical support during data collection. The mass spectrometry analysis of $A$. regius osteocalcin was performed at the Mass Spectrometry Facility, Department of Chemistry and Biochemistry, of the Howard Hughes Medical Institute (University of California at San Diego) and the Mass Spectrometry Service at Instituto de Tecnologia Química e Biológica (Oeiras, Portugal).

\section{REFERENCES}

1. Berman, H. M., Westbrook, J., Feng, Z., Gilliland, G., Bhat, T. N., Weissig, H., Shindyalov, I. N., and Bourne, P. E. (2000) Nucleic Acids Res. 28, 235-242.

2. Price, P. A., Otsuka, A. A., Poser, J. W., Kristaponis, J., and Raman, N. (1976) Proc. Natl. Acad. Sci. U.S.A. 73, 1447-1451.

3. Price, P. A., Poser, J. W., and Raman, N. (1976) Proc. Natl. Acad. Sci. U.S.A. 73, 3374-3375.

4. Cancela, M. L., Williamson, M. K., and Price, P. A. (1995) Int. J. Pept. Protein Res. 46, 419-423.

5. Simes, D. C., Williamson, M. K., Ortiz-Delgado, J. B., Viegas, C. S., Price, P. A., and Cancela, M. L. (2003) J. Bone Miner. Res. 18, 244-259.

6. Lian, J. B., Hauschka, P. V., and Gallop, P. M. (1978) Fed. Proc. 37, 2615-2620.

7. Price, P. A. (1985) Vitam. Horm. 42, 65-108.

8. Hauschka, P. V., Lian, J. B., Cole, D. E., and Gundberg, C. M. (1989) Physiol. Rev. 69, 990-1047.

9. Pinto, J. P., Ohresser, M. C., and Cancela, M. L. (2001) Gene 270, 77-91.

10. Poser, J. W., and Price, P. A. (1979) J. Biol. Chem. 254, 431436.

11. Poser, J. W., Esch, F. S., Ling, N. C., and Price, P. A. (1980) J. Biol. Chem. 255, 8685-8691.

12. Price, P. A. (1992) in Chemistry and biology of mineralized tissues (Slavkin, H., and Price, P. A., Eds.) pp 169-176, Elsevier Science Publishers, Amsterdam.

13. Viegas, C. S., Pinto, J. P., Conceicao, N., Simes, D. C., and Cancela, M. L. (2002) Gene 289, 97-107.

14. Colombo, G., Fanti, P., Yao, C., and Malluche, H. H. (1993) J. Bone Miner. Res. 8, 733-743.

15. Hauschka, P. V., and Carr, S. A. (1982) Biochemistry 21, 25382547.

16. Romberg, R. W., Werness, P. G., Riggs, B. L., and Mann, K. G. (1986) Biochemistry 25, 1176-1180.

17. Hunter, G. K., Hauschka, P. V., Poole, A. R., Rosenberg, L. C., and Goldberg, H. A. (1996) Biochem. J. 317 (Part 1), 59-64.

18. Malone, J. D., Teitelbaum, S. L., Griffin, G. L., Senior, R. M., and Kahn, A. J. (1982) J. Cell Biol. 92, 227-230.

19. Glowacki, J., Rey, C., Glimcher, M. J., Cox, K. A., and Lian, J. (1991) J. Cell. Biochem. 45, 292-302.

20. Lian, J. B., Tassinari, M., and Glowacki, J. (1984) J. Clin. Invest. $73,1223-1226$.

21. Liggett, W. H., Jr., Lian, J. B., Greenberger, J. S., and Glowacki, J. (1994) J. Cell. Biochem. 55, 190-199.

22. Ducy, P., Desbois, C., Boyce, B., Pinero, G., Story, B., Dunstan, C., Smith, E., Bonadio, J., Goldstein, S., Gundberg, C., Bradley, A., and Karsenty, G. (1996) Nature 382, 448-452.

23. Boskey, A. L., Gadaleta, S., Gundberg, C., Doty, S. B., Ducy, P., and Karsenty, G. (1998) Bone 23, 187-196.

24. Atkinson, R. A., Evans, J. S., Hauschka, P. V., Levine, B. A., Meats, R., Triffitt, J. T., Virdi, A. S., and Williams, R. J. (1995) Eur. J. Biochem. 232, 515-521.

25. Dowd, T. L., Rosen, J. F., Mints, L., and Gundberg, C. M. (2001) Biochim. Biophys. Acta 1535, 153-163.

26. Dowd, T. L., Rosen, J. F., Li, L., and Gundberg, C. M. (2003) Biochemistry 42, 7769-7779.

27. Chou, P. Y., and Fasman, G. D. (1978) Adv. Enzymol. Relat. Areas Mol. Biol. 47, 45-148.

28. Prigodich, R. V., O'Connor, T., and Coleman, J. E. (1985) Biochemistry 24, 6291-6298.

29. Isbell, D. T., Du, S., Schroering, A. G., Colombo, G., and Shelling, J. G. (1993) Biochemistry 32, 11352-11362.
30. Hoang, Q. Q., Sicheri, F., Howard, A. J., and Yang, D. S. C. (2003) Nature 425, 977-980.

31. Cancela, M. L., Ohresser, M. C., Reia, J. P., Viegas, C. S., Williamson, M. K., and Price, P. A. (2001) J. Bone Miner. Res. 16, 1611-1621.

32. Otwinowski, Z., and Minor, M. (1997) in Macromolecular Crystallography, Part A (Carter, C. W. J., and Sweet, R. M., Eds.) pp 307-336, Academic Press, New York.

33. Schneider, T. R., and Sheldrick, G. M. (2002) Acta Crystallogr. D58, 1772-1779.

34. Sheldrick, G. M. (2002) Z. Kristallogr. 217, 644-650.

35. Lamzin, V. S., and Wilson, K. S. (1993) Acta Crystallogr. D49, 129-147.

36. Collaborative Computational Project (1994) Acta Crystallogr. D50, 760-763.

37. Potterton, E., Briggs, P., Turkenburg, M., and Dodson, E. (2003) Acta Crystallogr. D59, 1131-1137.

38. Murshudov, N., Vagin, A. A., and Dodson, E. J. (1997) Acta Crystallogr. D53, 240-255.

39. Sheldrick, G. M., and Schneider, T. R. (1997) in Macromolecular Crystallography, Part B (Carter, C. W. J., and Sweet, R. M., Eds.) pp 319-343, Academic Press, New York.

40. Read, R. J. (1986) Acta Crystallogr. A42, 140-149.

41. McRee, D. E. (1992) J. Mol. Graphics 10, 44-46.

42. Merritt, E. A., and Bacon, D. J. (1997) Methods Enzymol. 277, 505-524.

43. Laskowski, R. A., MacArthur, M. W., Moss, D. S., and Thornton, J. M. (1993) J. Appl. Crystallogr. 26, 283-291.

44. Engh, R. A., and Huber, R. (1991) Acta Crystallogr. A47, 392400 .

45. Kraulis, P. J. (1991) J. Appl. Crystallogr. 24, 946-950.

46. Harding, M. M. (1999) Acta Crystallogr. D55, 1432-1443.

47. Harding, M. M. (2000) Acta Crystallogr. D56 (Part 7), 857-867.

48. Ravelli, R. B., and McSweeney, S. M. (2000) Struct. Folding Des. $8,315-328$.

49. Tickle, I. J., Laskowski, R. A., and Moss, D. S. (1998) Acta Crystallogr. D54, 547-557.

50. Nicholls, A., Sharp, S., and Honig, B. (1991) Proteins: Struct., Funct., Genet. 11, 281.

51. Sali, A., and Blundell, T. L. (1993) J. Mol. Biol. 234, 779-815.

52. Altschul, S. F., Madden, T. L., Schaffer, A. A., Zhang, J., Zhang, Z., Miller, W., and Lipman, D. J. (1997) Nucleic Acids Res. 25, 3389-3402.

53. Nishimoto, S. K., Waite, J. H., Nishimoto, M., and Kriwacki, R. W. (2003) J. Biol. Chem. 278, 11843-11848.

54. Cairns, J. R., and Price, P. A. (1994) J. Bone Miner. Res. 9, 19891997.

55. Price, P. A., Fraser, J. D., and Metz-Virca, G. (1987) Proc. Natl. Acad. Sci. U.S.A. 84, 8335-8339.

56. Berkner, K. L. (2000) J. Nutr. 130, 1877-1880.

57. Berkner, K. L., and Pudota, B. N. (1998) Proc. Natl. Acad. Sci. U.S.A. 95, 466-471.

58. Morris, D. P., Stevens, R. D., Wright, D. J., and Stafford, D. W. (1995) J. Biol. Chem. 270, 30491-30498.

59. Stanley, T. B., Humphries, J., High, K. A., and Stafford, D. W. (1999) Biochemistry 38, 15681-15687.

60. Benton, M. E., Price, P. A., and Suttie, J. W. (1995) Biochemistry $34,9541-9551$.

61. Kulman, J. D., Harris, J. E., Xie, L., and Davie, E. W. (2001) Proc. Natl. Acad. Sci. U.S.A. 98, 1370-1375.

62. Kulman, J. D., Harris, J. E., Haldeman, B. A., and Davie, E. W. (1997) Proc. Natl. Acad. Sci. U.S.A. 94, 9058-9062.

63. Di Scipio, R. G., Hermodson, M. A., Yates, S. G., and Davie, E. W. (1977) Biochemistry 16, 698-706.

64. Hagen, F. S., Gray, C. L., O’Hara, P., Grant, F. J., Saari, G. C., Woodbury, R. G., Hart, C. E., Insley, M., Kisiel, W., Kurachi, K., et al. (1986) Proc. Natl. Acad. Sci. U.S.A. 83, 2412-2416.

65. Hoskins, J., Norman, D. K., Beckmann, R. J., and Long, G. L. (1987) Proc. Natl. Acad. Sci. U.S.A. 84, 349-353.

66. Dahlback, B., Lundwall, A., and Stenflo, J. (1986) Proc. Natl. Acad. Sci. U.S.A. 83, 4199-4203.

67. Foster, D. C., Yoshitake, S., and Davie, E. W. (1985) Proc. Natl. Acad. Sci. U.S.A. 82, 4673-4677.

68. Manfioletti, G., Brancolini, C., Avanzi, G., and Schneider, C. (1993) Mol. Cell. Biol. 13, 4976-4985.

BI048336Z 